\title{
X-RAY STUDY OF CRAB-LIKE AND COMPOSITE SNRS
}

\author{
K. TORII, H. TSUNEMI \\ Osaka University \\ AND \\ P. SLANE \\ Harvard-Smithsonian Center for Astrophysics
}

\section{Introduction}

We have presented X-ray observations of Crab-like supernova remnants (SNRs) (plerions) and (non-thermal) composite SNRs. We have observed several objects including 3C58, CTA1, and G292.0+1.8. Since space is limited, here we summarize results on G292.0+1.8. For the other objects, please refer to each publication (Slane et al. 1997; Torii et al. 1997).

\section{G292.0+1.8 (MSH11-54)}

The composite nature of G292.0+1.8 has previously been in doubt. Although the center-filled radio morphology suggested an interior plerionic component, strong X-ray emission lines seemed to rule out such speculations. High resolution imaging with the Einstein HRI revealed its peculiar morphology (Tuohy et al. 1982) and X-ray emission was found to come from two distinct components, the central barlike feature and the ellipsoidal disk of approximately uniform surface brightness. Both the elemental abundances determined from X-ray spectra (Hughes et al. 1994) and the peculiar morphology suggested a Type II SN explosion.

We have found that the hard X-ray image (figure 1 right) shows a centerfilled compact nebula in contrast to the extended morphology in the soft band (figure 1 left). The position of the hard source coincides the radio "ridge" (Braun et al. 1986) and it is displaced to the east south-east direction from the apparent emission center. We interpret this component as a synchrotron nebula embedded in the shock heated thermal plasma. Since the X-ray morphology is far from a standard limb-brightened shell, possibly 
suggesting the presence of circumstellar material, we have applied a selfsimilar solution for a point explosion expanding into a non-uniform density (Sedov 1993), $\rho \sim r^{-2}$, corresponding to a constant velocity stellar wind of the progenitor. With a single component non ionization equilibrium model, the temperature is obtained as $k T \sim 0.8 \mathrm{keV}$. The age and the explosion energy are estimated to be $t_{\text {age }} \sim 4700 \pm 200 \mathrm{yr}$ and $E_{S N} \sim(1.25 \pm 0.07) \times 10^{50}$ ergs. Here we assumed the distance to the object of $4.8 \mathrm{kpc}$ (Saken et al. 1992). From the estimated age, we derive the projected velocity of the putative pulsar born at the explosion center. If we adopt a nominal expansion center obtained by optical observations (Braun et al. 1983) and assume that the putative pulsar is at the center of the compact nebula, we obtain a reasonable value (Lyne \& Lorimer 1994) for the velocity, $\mathrm{v}_{\text {trans }} \sim 790 \pm 320$ $d_{4.8 \mathrm{kpc}} t_{\mathrm{age}, 4700 \mathrm{yr}}^{-1} \mathrm{~km} \mathrm{~s}^{-1}$.
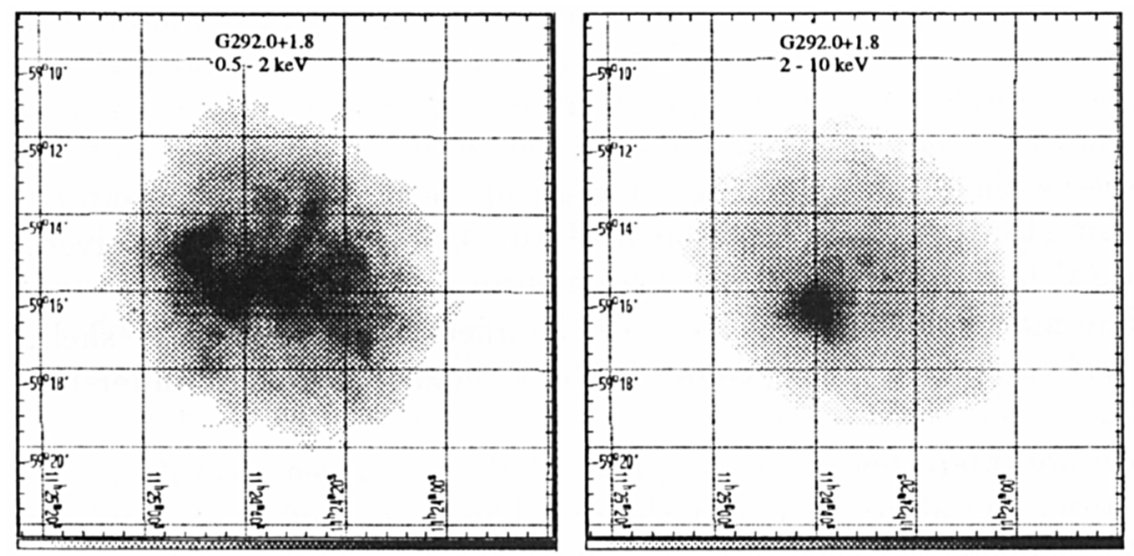

Figure 1. ASCA SIS images of G292.0+1.8. The images were deconvolved by the PSF of the telescope. Left: $0.5-2 \mathrm{keV}$, Right: $2-10 \mathrm{keV}$.

\section{References}

Braun, R., Goss, W.M., Danziger, I.J. and Boksenberg, A. (1983) Supernova Remnants and Their $X$-ray Emissions. Reidel, Dordrecht.

Braun, R., Goss, W.M. and Roger, R.S. (1986) $A \& A, 162,259$

Hughes, J.P. and Singh, K.P. (1994) $A p J, 422,126$

Lyne, A.G. and Lorimer, D.R. (1994) Nature, 369, 127

Saken, J.M., Fesen, R.A. and Shull, J.M. (1992) ApJS, 81, 715

Sedov, L.I. (1993) Similarity and Dimensional Methods in Mechanics 10th Edition. CRC Press, Boca Raton.

Slane, P., Seward, F.D., Bandiera, R., Torii, K. and Tsunemi, H. (1997) ApJ, 485, 221

Torii, K., Kinugasa, K., Hashimotodani, K., Tsunemi, H. and Slane, P.O. (1997) PASJ, submitted

Tuohy, I.R., Clark, D.H. and Burton, W.M. (1982) ApJL, 260, L65 